\title{
BMJ Open Epidemiology of placenta previa accreta: a systematic review and meta-analysis
}

\author{
Eric Jauniaux (D) , Lene Grønbeck, ${ }^{2}$ Catey Bunce (D) ," Jens Langhoff-Roos, ${ }^{4}$ \\ Sally L Collins ${ }^{5}$
}

To cite: Jauniaux E, Grønbeck L, Bunce C, et al. Epidemiology of placenta previa accreta: a systematic review and meta-analysis. BMJ Open 2019;9:e031193. doi:10.1136/ bmjopen-2019-031193

- Prepublication history and additional material for this paper are available online. To view please visit the journal (http:// dx.doi.org/10.1136/bmjopen2019-031193).

Received 21 April 2019 Revised 04 October 2019 Accepted 16 October 2019

Check for updates

(c) Author(s) (or their employer(s)) 2019. Re-use permitted under CC BY-NC. No commercial re-use. See rights and permissions. Published by BMJ.

${ }^{1}$ EGA Institute for Women Health, UCL, London, UK

${ }^{2}$ Department of Obstetrics, Rigshospitalet, University of Copenhagen, Kobenhavns, Denmark

${ }^{3}$ Primary Care and Public Health Sciences, King's College London, London, UK

${ }^{4}$ Departement of Obstetrics, Rigshospitalet, University of Copenhagen, Kobenhavn,

Denmark

${ }^{5}$ Nuffield Department of

Women's and Reproductive

Health, University of Oxford, Oxford, UK

Correspondence to

Professor Eric Jauniaux;

e.jauniaux@ucl.ac.uk

\section{ABSTRACT}

Objective To estimate the prevalence and incidence of placenta previa complicated by placenta accreta spectrum (PAS) and to examine the different criteria being used for the diagnosis.

Design Systematic review and meta-analysis.

Data sources PubMed, Google Scholar, ClinicalTrials.gov and MEDLINE were searched between August 1982 and September 2018.

Eligibility criteria Studies reporting on placenta previa complicated by PAS diagnosed in a defined obstetric population.

Data extraction and synthesis Two independent reviewers performed the data extraction using a predefined protocol and assessed the risk of bias using the Newcastle-0ttawa scale for observational studies, with difference agreed by consensus. The primary outcomes were overall prevalence of placenta previa, incidence of PAS according to the type of placenta previa and the reported clinical outcomes, including the number of peripartum hysterectomies and direct maternal mortality. The secondary outcomes included the criteria used for the prenatal ultrasound diagnosis of placenta previa and the criteria used to diagnose and grade PAS at birth.

Results A total of 258 articles were reviewed and 13 retrospective and 7 prospective studies were included in the analysis, which reported on 587 women with placenta previa and PAS. The meta-analysis indicated a significant $(p<0.001)$ heterogeneity between study estimates for the prevalence of placenta previa, the prevalence of placenta previa with PAS and the incidence of PAS in the placenta previa cohort. The median prevalence of placenta previa was $0.56 \%$ (IQR 0.39-1.24) whereas the median prevalence of placenta previa with PAS was $0.07 \%$ (IQR 0.05-0.16). The incidence of PAS in women with a placenta previa was $11.10 \%$ (IQR 7.65-17.35).

Conclusions The high heterogeneity in qualitative and diagnostic data between studies emphasises the need to implement standardised protocols for the diagnoses of both placenta previa and PAS, including the type of placenta previa and grade of villous invasiveness. PROSPERO registration number CRD42017068589

\section{INTRODUCTION}

Placenta accreta is a pathological condition of placentation associated with a high risk of massive obstetric haemorrhage during delivery. Initially described in 1937 by Irving and Hertig $^{1}$ as the abnormal adherence
Strengths and limitations of this study

- This study provides the first comprehensive evaluation of the epidemiology of placenta previa complicated by placenta accreta spectrum (PAS).

- The search was performed using predetermined eligibility criteria in a defined obstetric population.

- Thirteen out of 20 studies included in the study were retrospective limiting the overall quality of the analysis.

- Only six studies provided data on the prenatal ultrasound diagnosis of PAS in patients with placenta previa and nine studies on detailed histopathological findings.

- High level of inconsistency between estimates in prevalence and incidence did not allow for full metaanalysis of the clinical outcomes.

of the placenta to the myometrium due to the partial or complete absence of decidua basalis, it was subsequently redefined by Luke et $a l^{2}$ as a spectrum of abnormally adherent and invasive placentation disorders. Placenta accreta is now graded according to the depth of the villous penetration into the uterine wall starting with the abnormally adherent placenta or creta, where the villi attach directly to the surface of the myometrium without invading it, and extending to the invasive grades of placenta increta, where the villi penetrate deeply into the myometrium up to the uterine serosa, and placenta percreta, where the invasive villous tissue penetrates through the uterine serosa often entering the surrounding pelvic tissues. ${ }^{3-5}$ The different grades of the placenta accreta spectrum (PAS) can coexist in the same specimen and can be focal (just a small area of the placental bed) or extensive (including much of the placental bed). ${ }^{2}$

Over the last two decades, a growing body of epidemiology research has identified the effect of the rapid increase in caesarean delivery rates on the risks of PAS.$^{6-10}$ The main additional risk factor after a previous caesarean delivery is placenta previa. A large multicentric US cohort study noted that for women presenting 
with placenta previa and prior caesarean delivery, the risk of PAS was $3 \%, 11 \%, 40 \%, 61 \%$ and $67 \%$ for first, second, third, fourth and fifth or more caesarean deliveries, respectively. ${ }^{7}$ A national case-control study using the UK Obstetric Surveillance System found that the incidence of PAS increases from 1.7 per 10000 births overall to 577 per 10000 births in women with both a previous caesarean delivery and placenta previa. ${ }^{8}$

Both abnormal adherence and invasion of villous tissue into the myometrium result in failure of the placenta to separate spontaneously from the uterine wall at delivery. ${ }^{2-4}$ When unsuspected at the time of delivery, attempts to manually remove accreta villous tissue typically provoke rapid bleeding from the uteroplacental circulation. ${ }^{511} \mathrm{In}$ invasive cases, this can lead to massive obstetric haemorrhage due to the disruption of the deep uterine vasculature of the increta or percreta area. ${ }^{45}$ Not surprisingly, prenatal diagnosis of PAS has been shown to decrease maternal morbidity and mortality, and has thus become essential in improving its management. ${ }^{12} 13$ Tabsh et al were the first in 1982 to report on the prenatal ultrasound diagnosis of a case of placenta increta. ${ }^{14} \mathrm{~A}$ recent systematic review and meta-analysis of prenatal ultrasound diagnosis of placenta previa with PAS in women with a history of caesarean delivery has found that the overall diagnostic accuracy of ultrasound in specialist units is in $90.9 \% .^{15}$ However, in countries with well-established screening programmes for fetal anomalies, over half the cases of PAS are not diagnosed before delivery. ${ }^{810}$

Accreta placentation and in particular its invasive forms are impacting maternal health outcomes globally and its prevalence is likely to increase. Women with a history of previous caesarean delivery presenting with placenta previa complicated by PAS in an ongoing pregnancy are now the cohort of obstetric patients with the highest risk of delivery complications, ${ }^{16}$ however, their epidemiology has not been comprehensively reviewed yet. Health provision for the development of maternity centres with specialist teams, equipment, drugs, blood bank and intensive care infrastructure to safely manage women presenting with placenta previa and PAS requires an accurate evaluation of its epidemiology. The objective of this meta-analysis is to review the epidemiology of women presenting with placenta previa and to examine the different criteria used by the authors of cohort studies to diagnose placenta previa and PAS prenatally and to confirm the diagnosis of PAS at birth.

\section{MATERIALS AND METHODS}

A systematic review was undertaken of articles providing data on prevalence and incidence of PAS in women presenting with a placenta previa where the populations sampled were defined. PubMed, Google Scholar, ClinicalTrials.gov and MEDLINE were searched for studies published between the first prenatal ultrasound description of placenta accreta in August 1982 by Tabsh $e t a l^{4}$ and September 2018. The overall search strategy was inclusive of MeSH headings for the following terms "placenta accreta, placenta increta, placenta percreta, abnormally invasive placenta, morbidly adherent placenta and major placenta previa' (search strategy in online supplementary data 1 ). Title, abstracts and full text were independently assessed by the authors for content, data extraction and analysis. Additional relevant studies were identified from reference lists of reviews and editorials and by handsearching key journals and websites. All search results were combined in a reference database. Duplicates were removed by hand. The search was limited to articles published in English.

Two independent investigators (EJ and LG) selected studies in two stages. The abstracts of all potentially relevant papers were individually examined for suitability. Papers were only ruled out at this stage if they obviously did not meet the inclusion criteria. The remainders were obtained in full text and were independently assessed for content, data extraction and analysis. Disagreements between the two original reviewers were resolved by discussion with the third investigator (JL-R). Articles were excluded if; they were published before August 1982, contained no data on the study population such as the overall pregnancies, births and/or deliveries numbers, were case reports or were overlapping.

Study characteristics were extracted using a predesigned data extraction protocol including: author institution, year of publication, country of origin, study period, study type (retrospective, single institution, multiple institutions), total number of cases in the study population, type of placenta previa, diagnosis of PAS at birth (search strategy in online supplementary data 2). Outcome measures included the need to perform a peripartum hysterectomy and direct maternal mortality. Prior surgical history was also recorded. The reference standard for differential diagnosis between minor and major placenta previa was recorded based on the placental position inside the uterine cavity on transvaginal ultrasound with relation to the internal cervical os. For the diagnosis of accreta placentation, we referred to the clinical grading based on surgical findings at delivery as previously described ${ }^{17}$ and to histopathological findings when a caesarean hysterectomy was performed, that is, placental villi directly attached to the myometrium without interposing decidua or invading the uterine wall.

Two independent reviewers (EJ and LG) undertook the quality assessment with difference agreed by consensus. The Newcastle-Ottawa scale for observational studies was used to establish the risk of bias in selection (representativeness of the exposed cohort, ascertainment of exposure and the demonstration that the outcome of interest was not present at the start of the study), comparability (evaluation of the cohorts based on the design or analysis) and outcome assessment. ${ }^{18}$ These included retrospective versus prospective studies, single versus multiple institutions studies, prenatal ultrasound description of low-lying/placenta previa and PAS, histopathological confirmation of the diagnosis of the PAS and corresponding grade of invasiveness and detailed data on management and maternal outcomes. Studies that 
scored four stars for selection, two stars for comparability and three stars for ascertainment of the outcome were regarded to have a low risk of bias. Studies with two or three stars for selection, one for comparability and two for outcome ascertainment were considered to have a medium risk of bias. We deemed any study with a score of one for selection or outcome ascertainment, or zero for any of the three domains, to have a high risk of bias. No study was excluded based on the risk of bias assessment.

Analyses were conducted using STATA software (V.15; StataCorp). Standard Kurtosis analysis indicated that some values were not normally distributed and study specific estimates are therefore presented as median and IQR. A random-effects model was used to combine the studies while incorporating variations among studies unless there were three or less studies contributing to the meta-analysis in which case a fixed-effect model was used. Statistical heterogeneity was assessed with the Cochran's Q-test and the $\mathrm{I}^{2}$ statistic (the proportion of variation in study estimates because of heterogeneity rather than sampling error). Forest plots are presented to graphically summarise the study results and the pooled results. A test for heterogeneity between subgroups (ie, study types) was conducted.

\section{Patients and public involvement}

Patients and the public were not involved in the design or planning of the study.

\section{RESULTS}

The initial search provided 256 records with crossreferencing providing an additional two studies, making a total of 258 potentially relevant articles. After exclusion of duplicates and the two which were not available (figure 1), 220 remained. On screening the titles and abstracts, a further 162 were excluded as the reported outcomes were

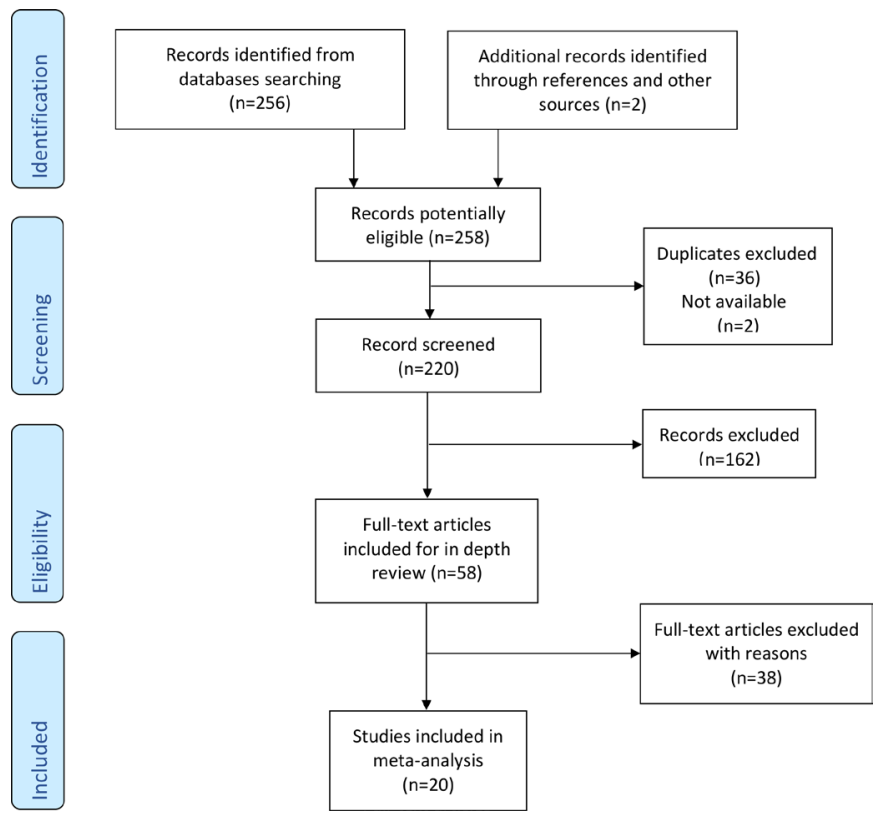

Figure 1 Flow diagram showing the selection of reports included in the review. not relevant, leaving 58 studies which were obtained for full text review. An additional 38 articles were excluded after full review including letters $(n=16)$, narrative reviews $(n=10)$ commentaries $(n=9)$, conference proceedings $(n=2)$ and duplication of data in another publication $(n=1)$, leaving 20 articles for the final analysis. ${ }^{19-38}$

There were 13 retrospective $19202325-27$ 29-31 33-35 38 and 7 prospective 21222428323637 studies including a total of 1207296 births and 23864 cases referred as pregnancies. There were 15 studies from a single institution $^{19-24}$ 27-30 32-34 3738 and 5 from multiple institutions ${ }^{25} 31$ or a geographical region. ${ }^{263536}$ Overall, 18 studies had low or medium risk of bias (full data in online supplementary data 3 ).

Table 1 presents the epidemiology data of the 20 studies. These studies included 587 women with placenta previa complicated by PAS out of 6628 cases of placenta previa. The median prevalence of placenta previa in the 20 studies was $0.56 \%$ (IQR 0.39-1.24) whereas the median prevalence of placenta previa with PAS was $0.07 \%$ (IQR $0.05-0.16)$. The median incidence of PAS in women with a placenta previa was $11.10 \%$ (IQR 7.65-17.35).

All authors except two ${ }^{29} 33$ reported on the criteria used for the prenatal ultrasound diagnosis of placenta previa. Six studies ${ }^{242630323738}$ only included major placenta previa in their cohort as defined as the placenta completely covering or partially covering the internal os of the cervix. The others included both major and minor placenta previa. The definition of minor placenta previa varied with two studies ${ }^{31}{ }^{36}$ using the placental edge being $<2 \mathrm{~cm}$ from the internal os, two studies using $<3 \mathrm{~cm}^{22} 2323$ and one study using $<3 \mathrm{~cm}$ or $<5 \mathrm{~cm}$ if associated with abnormal fetal presentation. ${ }^{21}$ The gestational age at confirmation of the prenatal diagnosis of placenta previa was reported in six studies ${ }^{22-24} 283237$ and ranged between 20 and 34 weeks and in one study the diagnosis of placenta previa was confirmed at birth when the placenta was found to be inserted in the lower segment. ${ }^{19}$

The ultrasound diagnostic signs for PAS were reported in six studies ${ }^{2428} 30323637$ with two studies also reporting on the use of MRI. ${ }^{2938}$ The clinical criteria used for the diagnosis of PAS at birth were reported by nine studies ${ }^{19} 2023272830333637$ and included a difficult delivery of the placenta without easy separation uterine wall or requiring a 'piecemeal removal' associated with heavy bleeding and excessive bleeding from the placental bed after placental delivery. One author described the presence of invasive villous tissue at delivery ${ }^{27}$ and one the need to suture the placental bed. ${ }^{23}$ None of the other authors reported on the gross appearance of the uterus or surgical findings at the time of caesarean delivery. In 12 studies, ${ }^{19} 232427-3133343637$ the prenatal and/or clinical diagnosis was confirmed by histopathological examination with detailed description of the microscopic criterion only reported in six. ${ }^{19} 2728303137$ Detailed histopathological findings on the depth of villous invasiveness were reported in 9 studies $^{2427-293133343637}$ out of the 20 studies (table 2). These included 283 cases of placenta previa accreta graded for $171(60.4 \%)$ as placenta accreta (adherent), 74 (26.2\%) 
Table 1 Prevalence of placenta previa with placenta accreta spectrum (PAS) per pregnancies or births in the corresponding obstetric population and incidence of PAS per cohorts of placenta previa

\begin{tabular}{|c|c|c|c|}
\hline References & Obstetric population & Prevalence $(\%)$ & Incidence (\%) \\
\hline Chattopadhyay et a $\left.\right|^{19}$ & 41206 births & $26(0.063)$ & 26/222 (11.7) \\
\hline Zaki et $\left.a\right|^{20}$ & 23070 births & $12(0.052)$ & $12 / 110(10.9)$ \\
\hline Ziadeh et $\left.a\right|^{21}$ & 18651 births & $13(0.070)$ & $13 / 65(20.0)$ \\
\hline Ghourab 22 & 18670 births & $11(0.059)$ & $11 / 138(8.0)$ \\
\hline Bahar et $\mathrm{al}^{23}$ & 42487 births & $53(0.125)$ & $53 / 306(17.3)$ \\
\hline Hamada et $a l^{24}$ & 2413 births & $5(0.207)$ & $5 / 70(7.1)$ \\
\hline Jang et $a l^{25}$ & 35030 births & $53(0.151)$ & $53 / 560(9.5)$ \\
\hline Rosenberg et $\mathrm{al}^{26}$ & 185476 births & $23(0.012)$ & $23 / 779(3.0)$ \\
\hline Kassem and Alzahrani ${ }^{27}$ & 29053 births & $25(0.085)$ & $25 / 122(20.5)$ \\
\hline Maher et $a^{28}$ & 24661 births & $42(0.170)$ & $42 / 577(7.3)$ \\
\hline Alchalabi et $\left.a\right|^{29}$ & 16845 births & $23(0.137)$ & $23 / 81(28.4)$ \\
\hline Asicioglu et $\left.a\right|^{30}$ & 112819 births & $46(0.041)$ & 46/364 (12.6) \\
\hline Sumigama et $a /^{31}$ & 96670 births & $46(0.048)$ & $46 / 954(4.8)$ \\
\hline Ahmed et $\left.a\right|^{32}$ & 3841 births & $14(0.365)$ & $14 / 5226.9$ \\
\hline Cheng and Lee ${ }^{33}$ & 81497 births & $39(0.048)$ & $39 / 921(4.2)$ \\
\hline Cho et $\left.a\right|^{34}$ & 11210 pregnancies & $39(0.348)$ & $39 / 442(8.8)$ \\
\hline Kollmann et $a^{35}$ & 218876 births & $13(0.006)$ & $13 / 328(4.0)$ \\
\hline Pilloni et $\left.a\right|^{36}$ & 108000 births & $37(0.034)$ & $37 / 314(11.8)$ \\
\hline Rezk and Shawky ${ }^{37}$ & 12654 pregnancies & $53(0.419)$ & $53 / 74(71.6)$ \\
\hline Wortman et a/38 & 148031 births & $14(0.010)$ & $14 / 157(8.9)$ \\
\hline
\end{tabular}

as placenta increta and $38(13.4 \%)$ as placenta percreta. These studies included a total of 383003 pregnancies or births and the prevalence for the different grades of placenta previa accreta was $0.05 \%, 0.02 \%$ and $0.01 \%$ for creta, increta and percreta, respectively.

The meta-analysis indicated statistically significant $(p<0.001)$ level of overall heterogeneity between study estimates for the prevalence of placenta previa (figure 2), the prevalence of placenta previa with PAS (figure 3) and the incidence of PAS in the placenta previa cohort (figure 4). There was strong evidence of inconsistency between study types with $\mathrm{I}^{2}$ values greater $85 \%$. The difference in heterogeneity between prospective versus retrospective studies was not statistically significantly $(\mathrm{p}=0.839)$ different (figure 2) whereas it was significant $(\mathrm{p}=0.014)$ for the prevalence of placenta previa accreta (figure 3). Adjusting for type of study (prospective vs retrospective) did not reduce inconsistency between studies. The in-between placenta previa major only versus minor and major placental previa was not significant $(\mathrm{p}=0.067)$ for

Table 2 Studies presenting detailed histopathological data on the depth of villous invasiveness (PAS grades)

\begin{tabular}{|c|c|c|c|c|}
\hline \multirow[b]{2}{*}{ References } & \multirow{2}{*}{$\begin{array}{l}\text { No of cases analysed/no of } \\
\text { cases included in the study }\end{array}$} & \multicolumn{3}{|c|}{ PAS grades } \\
\hline & & PC (\%) & PI (\%) & PP (\%) \\
\hline Hamada et $a l^{24}$ & $5 / 5$ & $3(60.0)$ & $2(40.0)$ & -- \\
\hline Maher et $a l^{28}$ & $42 / 42$ & $28(66.6)$ & $13(31.0)$ & $1(2.4)$ \\
\hline Achalabi et $a l^{29}$ & $23 / 23$ & $15(65.2)$ & $4(17.4)$ & $4(17.4)$ \\
\hline Cho et $a l^{34}$ & $39 / 39$ & $24(37.4)$ & $11(31.3)$ & 4 (31.3) \\
\hline Pilloni et $a l^{36}$ & $17 / 37$ & $7(41.2)$ & $4(23.5)$ & $6(35.3)$ \\
\hline Rezk and Shawky ${ }^{37}$ & $53 / 53$ & $31(58.5)$ & $14(26.4)$ & $8(15.1)$ \\
\hline Total & $283 / 309$ & $171(60.4)$ & $74(26.2)$ & 38 (13.4) \\
\hline
\end{tabular}

PAS, placenta accreta spectrum 


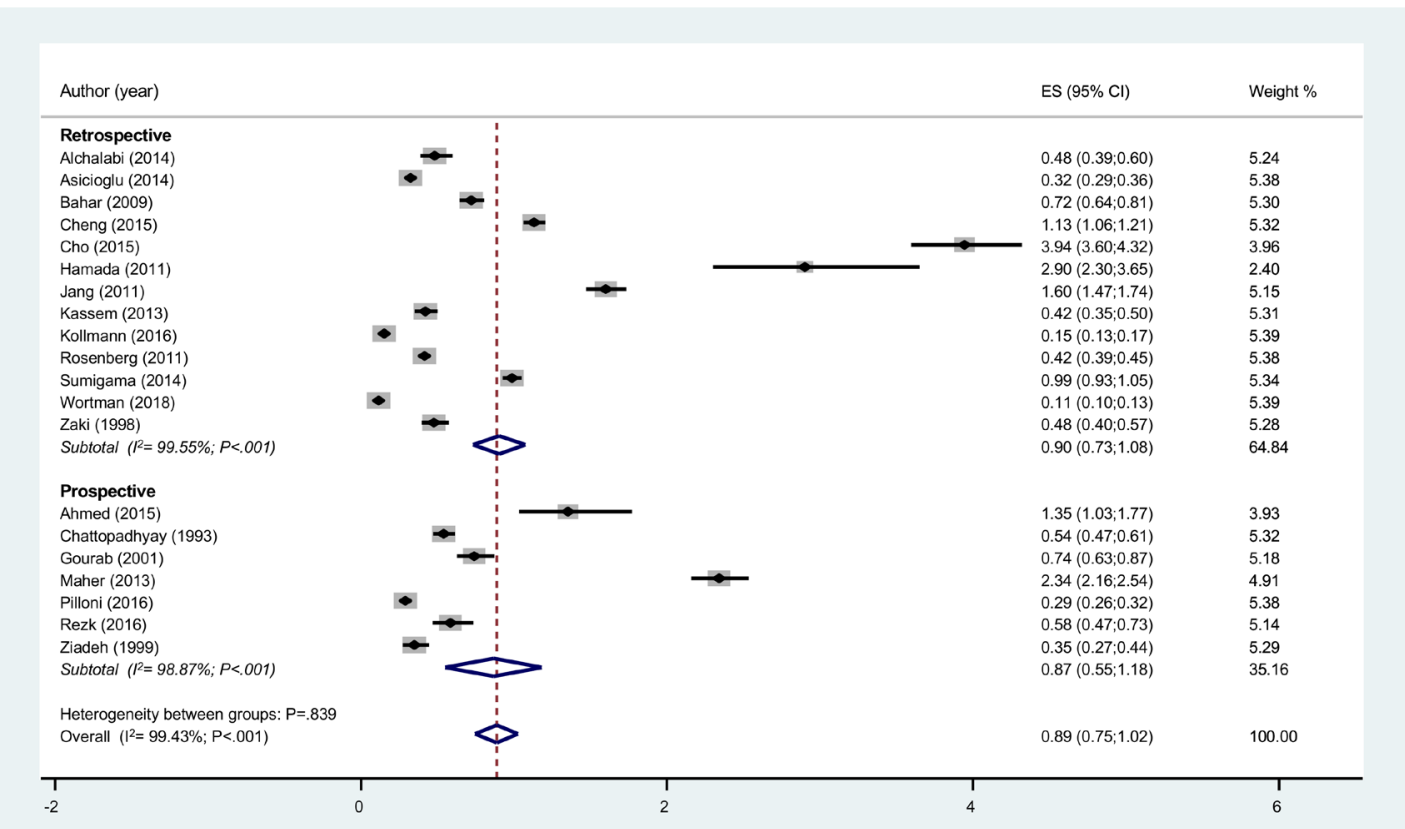

Figure 2 Forest plots showing the heterogeneity of prevalence data in prospective and retrospective cohort studies of women presenting with a placenta previa. Only first author's name is given for each reference. ES, effect size.

the incidence of PAS in patient with placenta previa (figure 4).

All authors but two ${ }^{22}$ reported on prior surgical history including caesarean section, ${ }^{19-21}{ }^{24-38}$ uterine curettage ${ }^{28}$ 30-32 343738 and myomectomy. ${ }^{283637}$ Data on surgical management were available in 14 out of the 20 studies $^{19} 2023$ 27-31 33-38 with 314 out of 441 women presenting with a placenta previa complicated by PAS. The median peripartum hysterectomy rate of $69.2 \%$ (IQR 50.0-84.0). Data on maternal mortality were available in 13 studies ${ }^{19-21} 232527-3032353738$ and PAS accounted for
5 maternal deaths ${ }^{19} 20252930$ out of 387 (1.3\%) cases of placenta previa with PAS.

\section{DISCUSSION}

This study provides a comprehensive evaluation of the prevalence of placenta previa complicated by PAS and the incidence of PAS in women presenting with a placenta previa. Women with a prior history of caesarean delivery presenting with a low-lying/placenta previa represent more than $90 \%$ of the cases of PAS. ${ }^{810} 16$ The

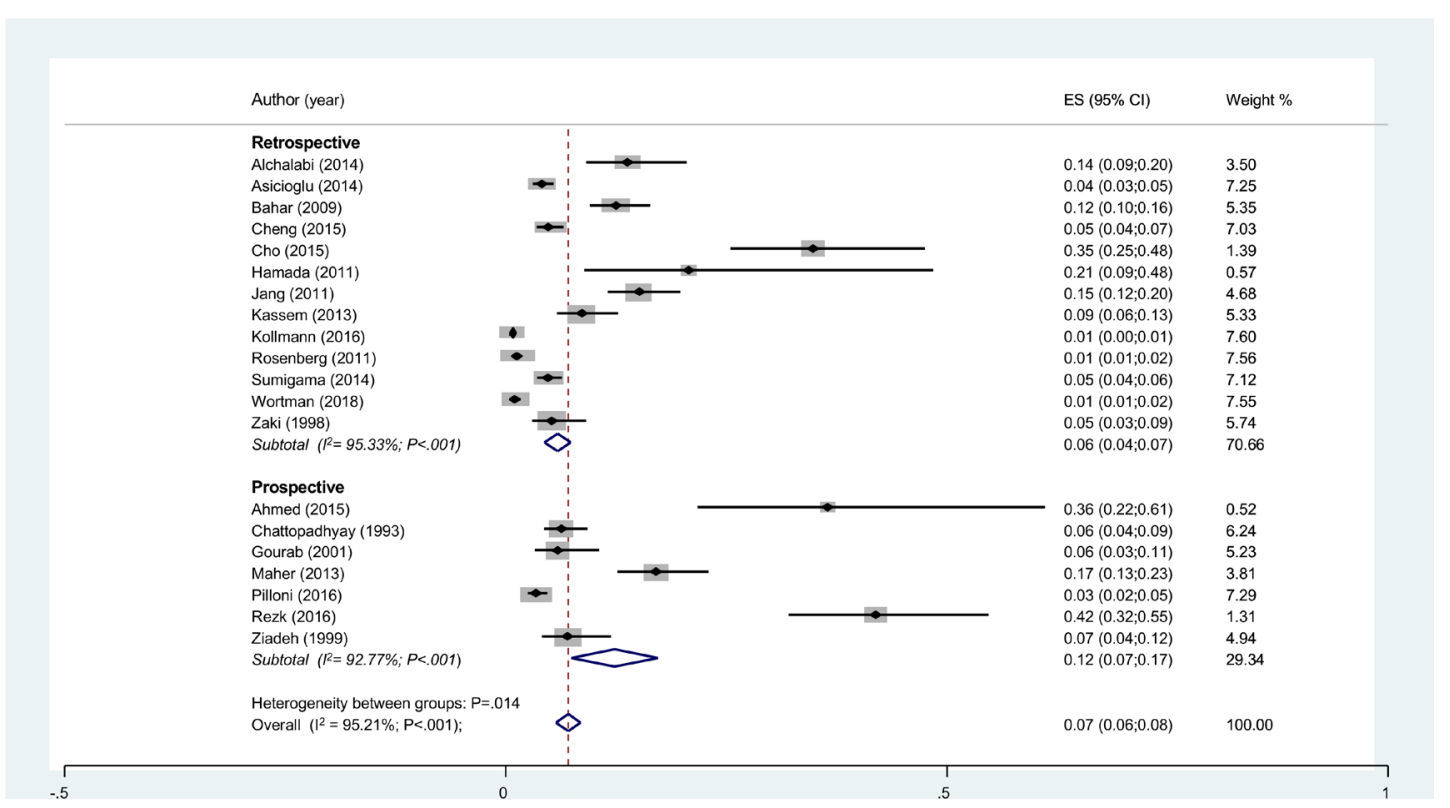

Figure 3 Forest plots showing heterogeneity in the prevalence data for prospective and retrospective cohort studies of women diagnosed with placenta previa accreta. Only first author's name is given for each reference. ES, effect size. 


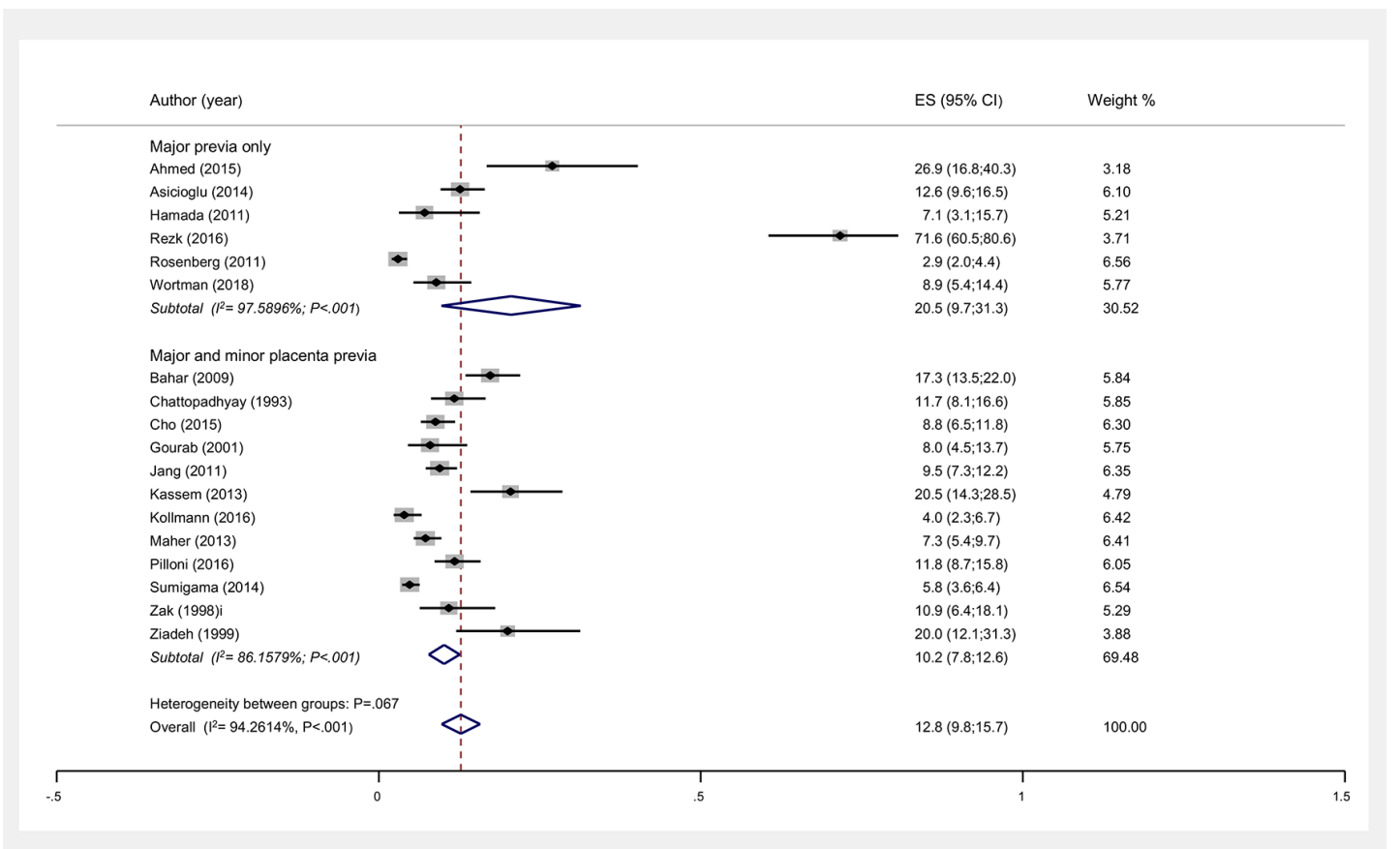

Figure 4 Forest plots showing the heterogeneity in cohort studies reporting incidence data for women diagnosed with placenta previa major and PAS and those with either placenta previa minor or major and PAS. ES, effect size.; PAS, placenta accreta spectrum.

meta-analysis indicates high heterogeneity for both the prenatal diagnosis of placenta previa and for the confirmation of the diagnosis of PAS at delivery. These findings highlight the need to use international standardised clinical protocols for the screening and management of this complex obstetric condition. The current situation limits the capacity building of healthcare providers on improvements in training, implementation of guidelines and changes in clinical practice behaviour.

Defining the position of the placenta inside the uterus was one of the first aims of obstetric ultrasound examination. ${ }^{39}{ }^{40}$ Following the development of real-time ultrasound imaging, placental location became an integral part of the mid-pregnancy ultrasound examination. ${ }^{41}$ Placenta previa was initially described with transabdominal scan as a placenta developing within the lower uterine segment and classified according to the relationship and/ or the distance between the lower placental edge and the internal os of the uterine cervix, that is, minor placenta previa when lower edge is inside the lower uterine segment down to the internal os and major placenta previa when the placenta covers the cervix. Minor placenta previa can be further subdivided into low-lying placenta when the lower edge does not reach the internal os and marginal placenta previa when it does. Major placenta previa can also be described as partial or complete depending on the amount of placental tissue covering the cervix. The use of transvaginal scanning has allowed for a more precise evaluation of the distance between the placental edge and the internal os ${ }^{42}{ }^{43}$ but as demonstrated in our metaanalysis, the reporting of the ultrasound criteria used for the diagnosis of placenta previa has been heterogeneous.
In addition, we found also wide variation in the gestational age at diagnosis. The timing of the confirmation of the diagnosis has a direct impact on epidemiology data as up to $70 \%$ of minor placenta previa at 20-23 weeks of gestation will resolve by $32-35$ weeks. ${ }^{44} 45$ An expert panel of the American Institute of Ultrasound in Medicine $^{46}$ has recently recommended ceasing the use of the terms 'partial' and 'marginal' and using the term 'placenta previa' only when the placenta lies directly over the internal os. The placenta should be reported as 'low-lying' when the placental edge is less than $2 \mathrm{~cm}$ from the internal os and as normal when the placental edge is more than $2 \mathrm{~cm}$ from the internal os. The findings of our meta-analysis highlight the need for the use of such a classification in further studies.

Only 6 of the 20 studies included in the present metaanalysis provided data on the prenatal ultrasound diagnosis of PAS in patients with placenta previa. We included in the systematic review all studies published since the first ultrasound description of PAS by Tabsh et al. ${ }^{14}$ We found no studies between 1982 and 1993 (table 1), which corresponds to the time when high-resolution grey-scale ultrasound imaging became widely available. Colour Doppler imaging was introduced for the diagnosis of PAS in $1992,{ }^{47}$ however, the sensitivity and specificity of greyscale imaging alone in diagnosing for placenta previa accreta are high when performed by the experience operators. ${ }^{15}$ These findings indicate that the prenatal diagnosis of PAS can be performed using standard ultrasound equipment. Unlike placenta previa which is routinely screened for at the time of the fetal anomaly scan, PAS is currently not screened for and the data available on 
the prenatal diagnosis of the condition come exclusively from specialist centres. ${ }^{16}$ In these centres, the diagnostic accuracy of ultrasound imaging is over $90 \%$, but similar to placenta previa, the description of the ultrasound signs used for the diagnosis of PAS has also been highly variable over the last two decades. ${ }^{48} 48$ The European Working Group on Abnormally Invasive Placenta and the Abnormally Invasive Placenta international expert group have recently proposed standardised descriptions of the ultrasound signs used for the prenatal diagnosis and a protocol for the ultrasound assessment of PAS. ${ }^{49}{ }^{50}$ The use of these protocols in prospective studies should also facilitate the screening of patients at high risk of PAS and in particular those with multiple prior caesarean deliveries presenting with a low-lying or placenta previa. ${ }^{51}$

We found significant heterogeneity in the qualitative definition and diagnosis of PAS at birth among the nine studies that provided a description of the clinical findings. ${ }^{19} 2023272830333637$ Only one of these studies described the invasive appearance of placental tissue at delivery ${ }^{27}$ whereas the others reported a difficult delivery of the placenta without easy separation from the uterine wall or requiring a 'piecemeal removal' associated with heavy bleeding as diagnostic of PAS. These clinical criteria were first described by Irving and Hertig ${ }^{1}$ in 1937 who did not have invasive cases in their cohort limiting their definition to abnormally adherent placenta and not to placenta increta or percreta. This definition also fails to clearly differentiate between abnormal adherence and placental retention as both present with similar clinical symptoms and aetiology ${ }^{52}$ leading to possible over diagnosis of placenta previa accreta. Similarly, the finding of excessive bleeding from the placental bed after delivery of the placenta is a common complication of nonaccreta placenta previa due to the implantation of the placenta in the lower uterine segment which contains less muscular fibres than the upper segment and is often thinner and dehiscent after multiple caesarean deliveries.

Detailed histopathological reports can only be obtained in those patients who have a hysterectomy or a partial myometrial resection and thus in many studies there is not histopathological confirmation of the clinical diagnosis. The main histological diagnostic criteria of accreta placentation, that is, absence of decidua between the tip of anchoring villi and the superficial myometrium, is found with increasing incidence with advancing gestation in pregnancies with no clinical evidence of PAS. ${ }^{5}$ Thus, the combination of clinical criteria that do not differentiate between placenta retention and adherent accreta and the use of non-diagnostic criteria of villous invasiveness may result in the overdiagnosis of the adherent grade of PAS (table 2), in particular in those studies reporting a low rate of caesarean hysterectomy. ${ }^{28}{ }^{36}$ Overall, this can explain the wide range in the prevalence $(0.04 \%-$ $0.42 \%)$ of placenta previa with PAS and incidence $(2.9 \%-$ $71.6 \%)$ of PAS in women presenting with placenta previa (figures 3 and 4).
Overall, management strategies and outcomes will vary depending on the accuracy of prenatal diagnosis, local surgical expertise and more recently access to a centre of excellence with multidisciplinary team approach. ${ }^{53}{ }^{54}$ In cases of high suspicion of PAS during caesarean delivery, $60 \%-70 \%$ of obstetricians-gynaecologists proceed with a peripartum hysterectomy ${ }^{556}$ By contrast with a conservative management approach, radical surgery is often considered to be safer, in particular in cases of invasive placentation. ${ }^{57}$ The association between a placenta previa and a PAS increases the risks of both maternal morbidity and mortality. In the present study, we found that a caesarean hysterectomy was the primary management option in around $70 \%$ of the patients presenting with a placenta previa and PAS. The interstudy range was wide with four studies ${ }^{19} 212937$ reporting peripartum hysterectomy rates $<50 \%$, five 2831323436 had rates between $50 \%$ and $99 \%$ and four ${ }^{22} 303538$ had rates of $100 \%$. This may be due to difference in study protocols, local expertise and the impact of prenatal diagnosis on management strategies but also as suggested by our analysis to difference in the rates of the different grades of PAS and the accuracy of clinical diagnosis at birth and detailed histopathological examination confirming the diagnosis.

The main limitations of this review are the quality of the published data. Thirteen out of 20 studies included in the analysis studies were retrospective and there was wide variation in the use of different ultrasound criteria for the prenatal diagnosis of placenta previa, in the clinical diagnosis of PAS at delivery and in the authors providing detailed histopathology data to confirm the clinical diagnosis. This is hampering the meta-analysis of the clinical outcomes in particular the incidence of major haemorrhage at delivery and the need and amount of blood transfusion but also the choice in management protocols and in particular the use of conservative management procedures. We would not, therefore, recommend the use of the pooled estimates beyond that of a support towards the development of standardised diagnostic protocols.

The prevalence of PAS in the general population of women giving birth varies widely. 8105859 A systematic review and meta-analysis of the prevalence of placenta praevia has found evidence suggestive of regional variation. ${ }^{60}$ As both conditions are often associated with prior caesarean sections, it is likely that national and local caesarean delivery rates, expertise in diagnosing both conditions antenatally and access to perinatal pathologist to confirm the diagnosis of PAS at birth will influence these epidemiology data. There is a need for further prospective multicentre studies with participatory methodologies involving local service providers and facility management to accurately evaluate the consequences of high caesarean sections rates on maternal health within a particular population. Within this context, accurate epidemiological data on PAS disorders are essential in planning screening programmes and in making provision for the development of centres of excellence for the management of this increasingly common complex 
obstetric condition. While the concept of core outcome measures within clinical trials is now well recognised and championed, greater efforts are required to disseminate this approach in epidemiological research to facilitate global estimation and recognition of problems emerging on a worldwide scale. Our study supports implementation, in both clinical practice and in reporting data on placenta previa accreta in the medical literature, of standardised protocols for prenatal diagnosis of both placenta previa and PAS, for the clinical diagnosis of PAS at birth and for the histopathological confirmation examination.

Contributors EJ, CB and JL-R contributed equally to the study design. EJ, LG and $\mathrm{JL}-\mathrm{R}$ collected the data and carried out the qualitative analysis. CB and EJ carried out the quantitative analysis. EJ, JL-R and SLC drafted the manuscript. All authors were involved in the critical discussion and approved this final version for publication. EJ is the guarantor of the study.

Funding The authors have not declared a specific grant for this research from any funding agency in the public, commercial or not-for-profit sectors.

Competing interests None declared.

Patient consent for publication Not required.

Provenance and peer review Not commissioned; externally peer reviewed.

Data availability statement Data are available on reasonable request.

Open access This is an open access article distributed in accordance with the Creative Commons Attribution Non Commercial (CC BY-NC 4.0) license, which permits others to distribute, remix, adapt, build upon this work non-commercially, and license their derivative works on different terms, provided the original work is properly cited, appropriate credit is given, any changes made indicated, and the use is non-commercial. See: http://creativecommons.org/licenses/by-nc/4.0/.

\section{ORCID iDs}

Eric Jauniaux http://orcid.org/0000-0003-0925-7737

Catey Bunce http://orcid.org/0000-0002-0935-3713

\section{REFERENCES}

1 Irving C, Hertig AT. A study of placenta accreta. surgery. Gynecol Obstet 1937;64:178-200.

2 Luke RK, Sharpe JW, Greene RR. Placenta accreta: the adherent or invasive placenta. Am J Obstet Gynecol 1966;95:660-8.

3 Jauniaux E, Jurkovic D. Placenta accreta: pathogenesis of a 20th century iatrogenic uterine disease. Placenta 2012;33:244-51.

4 Jauniaux E, Bhide A, Burton GJ. Pathophysiology of accreta. In: Silver R, ed. Placenta accreta syndrome. Portland: CRC Press, 2017: 13-28.

5 Jauniaux E, Collins S, Burton GJ. Placenta accreta spectrum: pathophysiology and evidence-based anatomy for prenatal ultrasound imaging. Am J Obstet Gynecol 2018;218:75-87.

6 Wu S, Kocherginsky M, Hibbard JU. Abnormal placentation: twentyyear analysis. Am J Obstet Gynecol 2005;192:1458-61.

7 Silver RM, Landon MB, Rouse DJ, et al. National Institute of child health and human development Maternal-Fetal medicine units network. maternal morbidity associated with multiple repeat cesarean deliveries. Obstet Gynecol 2006;107:1226-32.

8 Fitzpatrick KE, Sellers S, Spark P, et al. Incidence and risk factors for placenta accreta/increta/percreta in the UK: a national case-control study. PLoS One 2012;7:e52893.

9 Bowman ZS, Eller AG, Bardsley TR, et al. Risk factors for placenta accreta: a large prospective cohort. Am J Perinatol 2014;31:799-804.

10 Thurn L, Lindqvist PG, Jakobsson M, et al. Abnormally invasive placenta-prevalence, risk factors and antenatal suspicion: results from a large population-based pregnancy cohort study in the Nordic countries. BJOG: Int J Obstet Gy 2016;123:1348-55.

11 Silver RM, Branch DW, Spectrum PA. Placenta accreta spectrum. N Engl J Med 2018;378:1529-36.

12 Chantraine F, Braun T, Gonser M, et al. Prenatal diagnosis of abnormally invasive placenta reduces maternal peripartum hemorrhage and morbidity. Acta Obstet Gynecol Scand 2013;92:439-44.
13 Hall T, Wax JR, Lucas FL, et al. Prenatal sonographic diagnosis of placenta accreta-Impact on maternal and neonatal outcomes. J. Clin. Ultrasound 2014;42:449-55.

14 Tabsh KM. Brinkman Cr 3rd, King W. ultrasound diagnosis of placenta increta. J Clin Ultrasound 1982;10:288-90.

15 Jauniaux E, Bhide A. Prenatal ultrasound diagnosis and outcome of placenta previa accreta after cesarean delivery: a systematic review and meta-analysis. Am J Obstet Gynecol 2017;217:27-36.

16 Jauniaux E, Chantraine F, Silver RM, et al. Figo placenta accreta diagnosis and management expert consensus panel. FIGO consensus guidelines on placenta accreta spectrum disorders: epidemiology. Int J Gynaecol Obstet 2018;140:265-73.

17 Collins SL, Stevenson GN, Al-Khan A, et al. Three-Dimensional power Doppler ultrasonography for diagnosing abnormally invasive placenta and quantifying the risk. Obstetrics \& Gynecology 2015;126:645-53.

18 Wells GA, Shea B, O'Connell D, et al. The Newcastle-Ottawa scale (NOS) for assessing the quality of nonrandomised studies in meta-analyses, 2014. Available: www.ohri.ca/programs/clinical epidemiology/oxford.asp [Accessed 10 Jun 2018].

19 Chattopadhyay SK, Kharif H, Sherbeeni MM. Placenta praevia and accreta after previous caesarean section. Eur J Obstet Gynecol Reprod Biol 1993;52:151-6.

20 Zaki ZS, Bahar A, Ali M, et al. Risk factors and morbidity in patients with placenta previa accreta compared to placenta previa nonaccreta. Acta Obstet Gynecol Scand 1998;77:391-4.

21 Ziadeh SM, Abu-Heija AT, El-Jallad MF. Placental praevia and accreta: an analysis of two-years' experience. J Obstet Gynaecol 1999;19:584-6.

22 Ghourab S. Third-trimester transvaginal ultrasonography in placenta previa: does the shape of the lower placental edge predict clinical outcome? Ultrasound Obstet Gynecol 2001;18:103-8.

23 Bahar A, Abusham A, Eskandar M, et al. Risk factors and pregnancy outcome in different types of placenta previa. Journal of Obstetrics and Gynaecology Canada 2009;31:126-31.

24 Hamada S, Hasegawa J, Nakamura M, et al. Ultrasonographic findings of placenta lacunae and a lack of a clear zone in cases with placenta previa and normal placenta. Prenat Diagn 2011;31:1062-5.

25 Jang DG, We JS, Shin JU, et al. Maternal outcomes according to placental position in placental previa. Int J Med Sci 2011;8:439-44.

26 Rosenberg T, Pariente G, Sergienko R, et al. Critical analysis of risk factors and outcome of placenta previa. Arch Gynecol Obstet 2011;284:47-51.

27 Kassem GA, Alzahrani A. Maternal and neonatal outcomes of placenta previa and placenta accreta: three years of experience with a two-consultant approach. Int J Womens Health 2013;28:803-10.

28 Maher MA, Abdelaziz A, Bazeed MF. Diagnostic accuracy of ultrasound and $\mathrm{MRI}$ in the prenatal diagnosis of placenta accreta. Acta Obstet Gynecol Scand 2013;92:1017-22.

29 Alchalabi Haifa'a, Lataifeh I, Obeidat B, et al. Morbidly adherent placenta previa in current practice: prediction and maternal morbidity in a series of 23 women who underwent hysterectomy. J Matern Fetal Neonatal Med 2014;27:1734-7.

30 Asıcıoglu O, Şahbaz A, Güngördük K, et al. Maternal and perinatal outcomes in women with placenta praevia and accreta in teaching hospitals in Western turkey. J Obstet Gynaecol 2014;34:462-6.

31 Sumigama S, Sugiyama C, Kotani T, et al. Uterine sutures at prior caesarean section and placenta accreta in subsequent pregnancy: a case-control study. BJOG: Int J Obstet Gy 2014;121:866-75.

32 Ahmed SR, Aitallah A, Abdelghafar HM, et al. Major placenta previa: rate, maternal and neonatal outcomes experience at a tertiary MaternityHospital, Sohag, Egypt: a prospective study. J Clin Diagn Res 2015;9:17-19.

33 Cheng KK, Lee MM. Rising incidence of morbidly adherent placenta and its association with previous caesarean section: a 15-year analysis in a tertiary hospital in Hong Kong. Hong Kong Med J 2015;21:511-7.

34 Cho HY, Hwang HS, Jung I, et al. Diagnosis of placenta accreta by uterine artery Doppler velocimetry in patients with placenta previa. $J$ Ultrasound Med 2015;34:1571-5.

35 Kollmann M, Gaulhofer J, Lang U, et al. Placenta praevia: incidence, risk factors and outcome. J Matern Fetal Neonatal Med 2016;29:1395-8.

36 Pilloni E, Alemanno MG, Gaglioti P, et al. Accuracy of ultrasound in antenatal diagnosis of placental attachment disorders. Ultrasound Obstet Gynecol 2016;47:302-7.

37 Rezk MA-A, Shawky M. Grey-Scale and colour Doppler ultrasound versus magnetic resonance imaging for the prenatal diagnosis of placenta accreta. The Journal of Maternal-Fetal \& Neonatal Medicine 2016;29:218-23. 
38 Wortman A, Schaefer S, Mclntire D, et al. Complete placenta previa: ultrasound biometry and surgical outcomes. AJP Rep 2018;08:e74-8

39 Gottesfeld KR, Thompson HE, Holmes JH, et al. Ultrasonic placentography--a new method for placental localization. Am J Obstet Gynecol 1966;96:538-47.

40 Kohorn El, Walker RH, Morrison J, et al. A comparison between ultrasonic compound $\mathrm{B}$ scanning and radioisotope scanning. Am J Obstet Gynecol 1969;103:868-77.

41 Ballas S, Gitstein S, Jaffa AJ, et al. Midtrimester placenta previa: normal or pathologic finding. Obstetrics \& Gynecology 1979;54:12-14.

42 Leerentveld RA, Gilberts ECAM, Arnold MJCWJ, et al. Accuracy and safety of transvaginal sonographic placental localization. Obstetrics \& Gynecology 1990;76:759-62.

43 Smith RS, Lauria MR, Comstock $\mathrm{CH}$, et al. Transvaginal ultrasonography for all placentas that appear to be low-lying or over the internal cervical os. Ultrasound Obstet Gynecol 1997;9:22-4.

44 Dashe JS, Mclntire DD, Ramus RM, et al. Persistence of placenta previa according to gestational age at ultrasound detection. Obstet Gynecol 2002;99:692-7.

45 Quant HS, Friedman AM, Wang E, et al. Transabdomina ultrasonography as a screening test for second-trimester placenta previa. Obstetrics \& Gynecology 2014;123:628-33.

46 Reddy UM, Abuhamad AZ, Levine D, et al. Fetal imaging: Executive summary of a joint Eunice Kennedy Shriver National Institute of child health and human development, Society for MaternalFetal medicine, American Institute of ultrasound in medicine, American College of obstetricians and Gynecologists, American College of radiology, Society for pediatric radiology, and society of radiologists in ultrasound fetal imaging workshop. J Ultrasound Med 2014;33:745-57.

47 Jauniaux E, Collins SL, Jurkovic D, et al. Accreta placentation: a systematic review of prenatal ultrasound imaging and grading of villous invasiveness. Am J Obstet Gynecol 2016;215:712-21.

48 Collins SL, Chantraine F, Morgan TK, et al. Abnormally adherent and invasive placenta: a spectrum disorder in need of a name. Ultrasound Obstet Gynecol 2018;51:165-6.

49 Collins SL, Ashcroft A, Braun T, et al. Proposal for standardized ultrasound descriptors of abnormally invasive placenta (AIP). Ultrasound Obstet Gynecol 2016;47:271-5
50 Alfirevic Z, Tang A-W, Collins SL, et al. Palacios-Jaraquemadas, on behalf of the ad-hoc international AIP expert group. pro Forma for ultrasound reporting in suspected abnormally invasive placenta (AIP); an international consensus. Ultrasound Obstet Gynecol 2016;47:276-8.

51 Jauniaux E, Bhide A, Kennedy A, et al. Figo placenta accreta diagnosis and management expert consensus panel. FIGO consensus guidelines on placenta accreta spectrum disorders: prenatal diagnosis and screening. Int J Gynaecol Obstet 2018;140:274-80.

52 Greenbaum S, Wainstock T, Dukler D, et al. Underlying mechanisms of retained placenta: evidence from a population based cohort study. Eur J Obstet Gynecol Reprod Biol 2017;216:12-17.

53 Obstetric care consensus No. 7 summary: placenta accreta spectrum. Obstet Gynecol 2018;132:1519-21.

54 Bartels HC, Rogers AC, O'Brien D, et al. Association of implementing a multidisciplinary team approach in the management of morbidly adherent placenta with maternal morbidity and mortality. Obstet Gynecol 2018;132:1167-76.

55 Wright JD, Silver RM, Bonanno C, et al. Practice patterns and knowledge of obstetricians and gynecologists regarding placenta accreta. J Matern Fetal Neonatal Med 2013;26:1602-9.

56 Cal M, Ayres-de-Campos D, Jauniaux E. International survey of practices used in the diagnosis and management of placenta accreta spectrum disorders. Int J Gynecol Obstet 2018;140:307-11.

57 Allen L, Jauniaux E, Hobson S, et al. Figo placenta accreta diagnosis and management expert consensus panel. FIGO consensus guidelines on placenta accreta spectrum disorders: nonconservative surgical management. Int J Gynaecol Obstet 2018;140:281-90.

58 Farquhar CM, Li Z, Lensen S, et al. Incidence, risk factors and perinatal outcomes for placenta accreta in Australia and New Zealand: a case-control study. BMJ Open 2017;7:e017713.

59 Sargent W, Collins SL. Are women antenatally diagnosed with abnormally invasive placenta receiving optimal management in England? an observational study of planned place of delivery. Acta Obstet Gynecol Scand 2019;98:337-41.

60 Cresswell JA, Ronsmans C, Calvert C, et al. Prevalence of placenta praevia by world region: a systematic review and meta-analysis. Trop Med Int Health 2013;18:712-24. 